\title{
A Nanomechanical Investigation of Engineered Bone Tissue Comparing Elastoplastic and Viscoelastoplastic Modeling
}

\author{
Marco Boi, ${ }^{1}$ Gregorio Marchiori, ${ }^{1}$ Maria Sartori, ${ }^{2}$ Francesca Salamanna, ${ }^{3}$ \\ Gabriela Graziani, ${ }^{1}$ Alessandro Russo, ${ }^{1}$ Andrea Visani, ${ }^{4}$ Mauro Girolami, ${ }^{5}$ \\ Milena Fini, ${ }^{3}$ and Michele Bianchi ${ }^{1}$ \\ ${ }^{1}$ Rizzoli Orthopaedic Institute, NanoBiotechnology Laboratory (NaBi), Research Innovation and Technology Department (RIT), \\ Via di Barbiano 1/10, 40136 Bologna, Italy \\ ${ }^{2}$ Rizzoli Orthopaedic Institute, Laboratory of Biocompatibility, Technological Innovations and Advanced Therapies, \\ Research Innovation and Technology Department (RIT), Via di Barbiano 1/10, Bologna, Italy \\ ${ }^{3}$ Rizzoli Orthopaedic Institute, Laboratory of Preclinical and Surgical Studies, 40136 Bologna, Italy \\ ${ }^{4}$ Rizzoli Orthopaedic Institute, Laboratory of Biomechanics and Technology Innovation, Via di Barbiano 1/10, \\ 40136 Bologna, Italy \\ ${ }^{5}$ Rizzoli Orthopaedic Institute, Orthopedics and Traumatology Department, Via Pupilli 1, \\ 40010 Bologna, Italy
}

Correspondence should be addressed to Marco Boi; m.boi@biomec.ior.it

Received 19 June 2017; Accepted 25 July 2017; Published 27 August 2017

Academic Editor: Renal Backov

Copyright (C) 2017 Marco Boi et al. This is an open access article distributed under the Creative Commons Attribution License, which permits unrestricted use, distribution, and reproduction in any medium, provided the original work is properly cited.

It is common practice to implement the elastoplastic Oliver and Pharr (OP) model to investigate the spatial and temporal variations of mechanical properties of engineered bone. However, the viscoelastoplastic (VEP) model may be preferred being envisaged to provide additional insights into the regeneration process, as it allows evaluating also the viscous content of bone tissue. In this work, the elastic modulus $\left(E_{R}\right)$, contact hardness $\left(H_{C}\right)$, hardness $(H)$, and viscosity index $\left(\eta_{Q}\right)$ of newly formed bone tissue regenerated at 4 and 12 weeks from the implantation of a macroporous hydroxyapatite scaffold in a rabbit femoral critical-size model were addressed and compared to the mechanical properties of preexisting bone. Indentation curves were fitted with both the OP and VEP models. The VEP model outlined a wider gap between the mechanical properties of native and regenerated tissue when compared to the OP model. In addition, the VEP model indicated an increase of the viscosity index from 4 to 12 weeks, supporting the evidence of a still active regeneration process. The reported results confirmed the higher ability of VEP model compared to the more diffused OP model to provide important insights into bone mechanical properties, also during the bone regeneration process.

\section{Introduction}

Bone tissue is a highly anisotropic, heterogeneous, and viscoelastic material exhibiting a complex hierarchical structure at multiple length scales $[1,2]$. Conventional macroscopic mechanical tests are not able to detect the mechanical properties at the trabecula or osteon level $[3,4]$. On the other hand, instrumented indentation (or "nanoindentation") is a widely accepted method to investigate the mechanical properties of bone tissue at the micro- and nanoscale [5-7].
In bone engineering context, it is still common practice to utilize the well-known Oliver and Pharr (OP) model [8] to analyze indentation data in terms of elastic/plastic contribution, which however overlooks the viscous behavior of bone tissue [9].

During the last decade, Oyen's group developed and validated a viscoelastic-plastic (VEP) model that foresees a three-step consecutive fitting of a loading-creep-unloading curve in order to extract the main mechanical parameters of the viscoelastic material such as elastic modulus $\left(E_{R}\right)$, contact 
hardness $\left(H_{C}\right)$, hardness $(H)$, and viscosity index $\left(\eta_{Q}\right)[10,11]$. The hypothesis is that the VEP model can provide additional details over bone maturation process not directly accessible through the common OP analysis. However, to date, the application of the VEP model has been almost exclusively limited to the analysis of native (i.e., not engineered) bone tissue, whereas reports of VEP analysis of bone mechanical properties during tissue regeneration are lacking.

Therefore, here we compared the ability of OP and VEP models to catch the evolution of mechanical properties of bone tissue during the regenerative process, by analyzing the mechanical characteristics of preexistent and newly formed bone tissue regenerated at 4 and 12 weeks from the implantation of a macroporous hydroxyapatite (HA) scaffold in a preclinical model of critical-size defect.

\section{Materials and Methods}

2.1. Scaffold Synthesis. A commercial HA powder (Finceramica Faenza Spa, Italy) was calcined at $1000^{\circ} \mathrm{C}$ for 2 hours in order to decrease the specific surface area value and prepare stable suspensions with higher solid loading. An optimized suspension was prepared by ball mixing deionized water, $1.5 \mathrm{wt} \%$ of dispersant Dolapix CE-64 (Zschimmer \& Schwarz, Lahnstein, Germany) having a specific surface area value of $3.93 \mathrm{~m}^{2} / \mathrm{g}$. After 8 hours, when a homogeneous slurry was obtained, $1.4 \mathrm{wt} \%$ of foaming agent (Dermocin BS, Fratelli Ricci, Italia) was added. After 12 hours, the foamed slurry was casted in water draining moulds, allowing the setting of the foamed system, and finally dried in air at room temperature for 48 hours. The thermal treatment has been performed in atmosphere in Ar at a sintering temperature of $1200^{\circ} \mathrm{C}$ for $1 \mathrm{~h}$.

2.2. In Vivo Study and Sample Preparation. Macroporous HA scaffolds $(6 \times 8 \mathrm{~mm}, 90 \%$ of porosity, ISTEC-CNR, Faenza, Italy) were implanted in the femoral condyle of six male rabbits (New Zealand White, 15 weeks of age), in accordance with the European and Italian Law on animal experimentation and after the approval of the research protocol by the Ethical Committee of Rizzoli Orthopaedic Institute and by the public authorities as provided by Law by Decree $116 / 92$. Animals were housed at a controlled temperature of $22 \pm 1{ }^{\circ} \mathrm{C}$ and relative humidity of $55 \pm 5 \%$ in single boxes and fed a standard diet (Mucedola, Milano, Italy) with filtered tap water ad libitum. At the time of surgery, general anesthesia was induced with an intramuscular injection of $44 \mathrm{mg} / \mathrm{kg}$ ketamine (Farmaceutici Gellini SpA, Latina, Italy) and $3 \mathrm{mg} / \mathrm{kg}$ xylazine (Bayer AG, Leverkusen, Germany) under assisted ventilation with $\mathrm{O}_{2} /$ air $\left(1 / 0.4 \mathrm{lmin}^{-1}\right)$ mixture and $2.5 \%$ isoflurane (Forane, Abbot $\mathrm{SpA}$, Latina, Italy). After having shaved and disinfected the posterior legs, longitudinal incisions on the lateral surface of femoral condyles were made to expose the distal femurs. Critical-size defects of $6 \mathrm{~mm}$ in diameter and $8 \mathrm{~mm}$ in depth were created with a low speed drill, adopting drills with increasing diameter up to $6 \mathrm{~mm}$ and flushing and cooling the defects with a profuse irrigation with cold sterile $0.9 \% \mathrm{NaCl}$ solution to remove bone debris and to prevent the risk of bone necrosis. Subsequently, HA scaffolds were implanted by press-fit in one of the two femoral condyles and wounds were sutured in layers. Postoperatively antibiotics and analgesics were administered and after 4 and 12 weeks animals ( 3 for each experimental time) were pharmacologically euthanized with an intravenous administration of Tanax (Hoechst, Frankfurt am Main, Germany) under general anesthesia. Femoral condyles were excised, stripped of soft tissue, and macroscopically evaluated for the presence of hematomas, oedema, and inflammatory tissue reactions. Bone segments were fixed in $4 \%(\mathrm{v} / \mathrm{v})$ buffered paraformaldehyde, dehydrated in a graded series of alcohol/water mixture until the absolute to remove the water from the specimens, and finally embedded in polymethyl methacrylate (PMMA) resin. For nanoindentation tests, the embedded samples were sectioned along a plane transversal to the long axis of the implant and the obtained histological slides were polished using silicon carbide paper (from 800 to 2500 grits $)$ and alumina slurry $(0.05 \mu \mathrm{m}$ grit $)$. Sections for histological analyses (thickness $\sim 10 \mu \mathrm{m}$ ) were stained with Toluidine Blue, Acid Fuchsin, and Fast Green.

2.3. Nanomechanical Testing. Nanoindentation tests were carried out using a $\mathrm{NHT}^{2}$ nanoindentation tester (CSMInstruments, Anton Paar, Peseux, Switzerland), equipped with a diamond Berkovich tip, on both engineered (i.e., newly formed) and preexistent tissue (Figure 1).

Three condyles for each experimental time (i.e., one condyle for each animal) have been investigated. Within each condyle, at least 5 regions of interest (ROIs) were selected with the help of an optical microscope (with $5 x, 20 x$, and 50x objectives Olympus M Plan N) connected to the indenter head. From 10 to 15 indents were acquired within each ROI using two-dimensional $(2 \times 2)$ matrices of indents keeping the distance among subsequent indents as $25 \mu \mathrm{m}$ in both $x$ and $y$ directions (Figure 2(a)). All indentations were acquired following a protocol adapted from [12]: briefly, a trapezoidal load control profile was drawn, with maximum load $\left(P_{\text {MAX }}\right)$ of $8 \mathrm{mN}$ and holding time of $30 \mathrm{~s}$ (time creep, $t_{C}$ ); loading and unloading time were $10 \mathrm{~s}\left(t_{R}\right)$. All the indents found on osteocyte lacunae or close (below $\sim 15 \mu \mathrm{m}$ ) to PMMA and scaffold were removed from the dataset [13].

\section{Data Analysis}

3.1. The Oliver and Pharr (OP) Model. The OP model allows determining the reduced elastic modulus $\left(E_{R}\right)$ and contact hardness $\left(H_{C}\right)$ through a linear fitting of the unloading curve by assuming a purely elastic material response during unloading. In order to limit the contribute of the timedependent component of bone tissue, a rapid unloading rate [14] and an extended creep time [15] are commonly used when applying the OP model to viscoelastic materials. Stiffness $(S)$ is calculated as the slope of the unloading curve at the peak load; in this study, $S$ was calculated from the slope of the unloading curve in the region $40-98 \%$ of the maximum load. The Berkovich indenter tip was calibrated with a fused quartz reference sample (certificated plane strain modulus $\left.E^{*}=75.1 \pm 0.4 \mathrm{GPa}\right)$ using a calibration intensive mode (110 indentations using 22 different loads, from $0.1 \mathrm{mN}$ to $100 \mathrm{mN}$ ) in order to obtain the projected contact area $\left(A_{C}\right)$ from the 


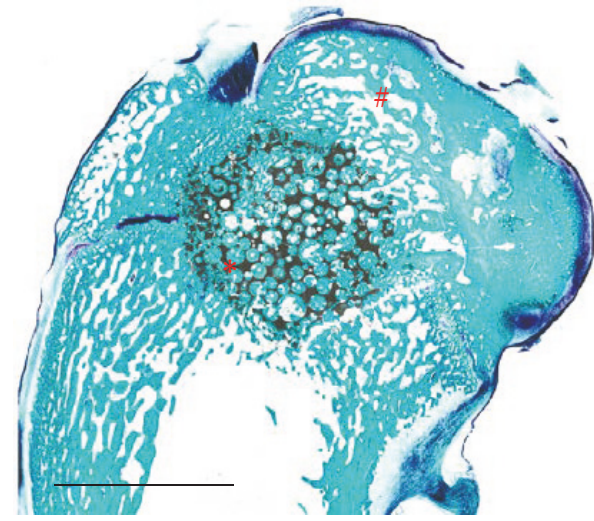

(a)

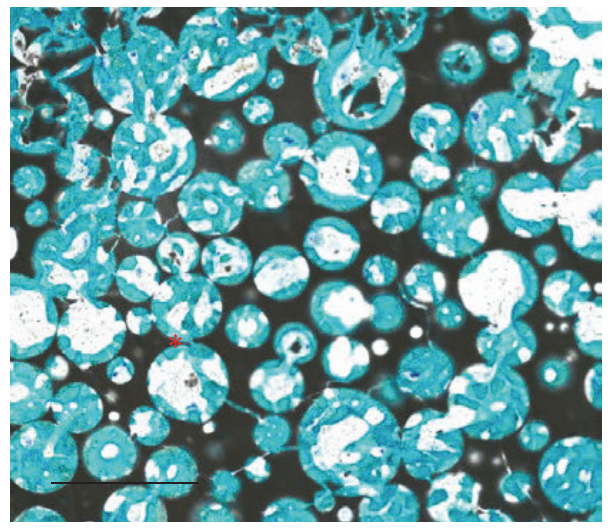

(c)

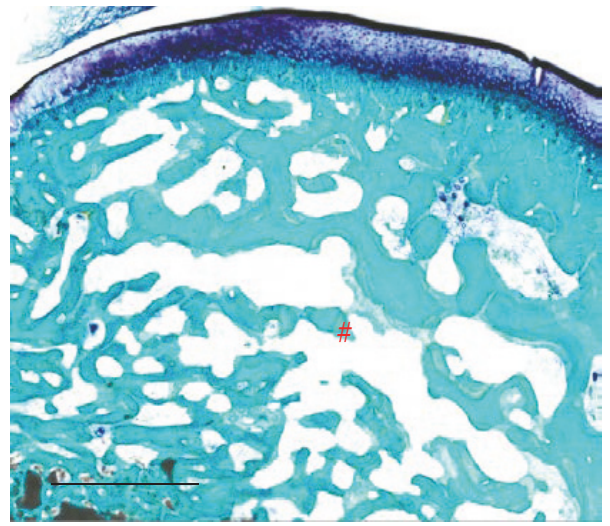

(e)

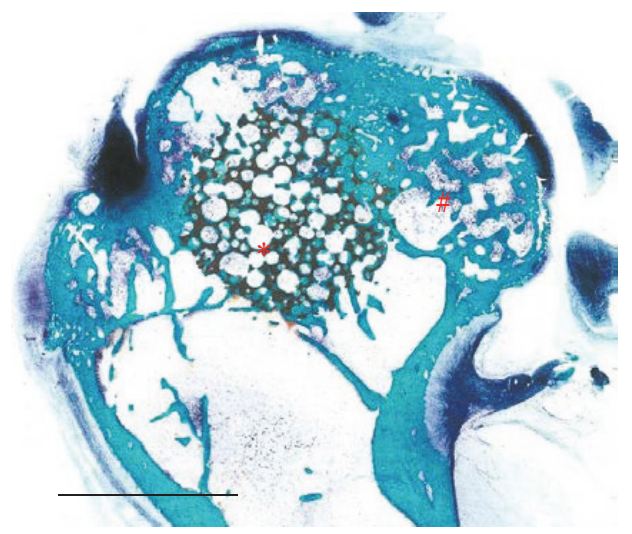

(b)

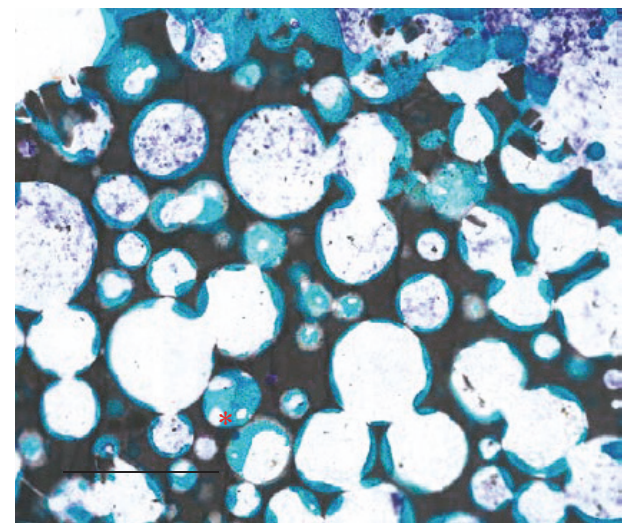

(d)

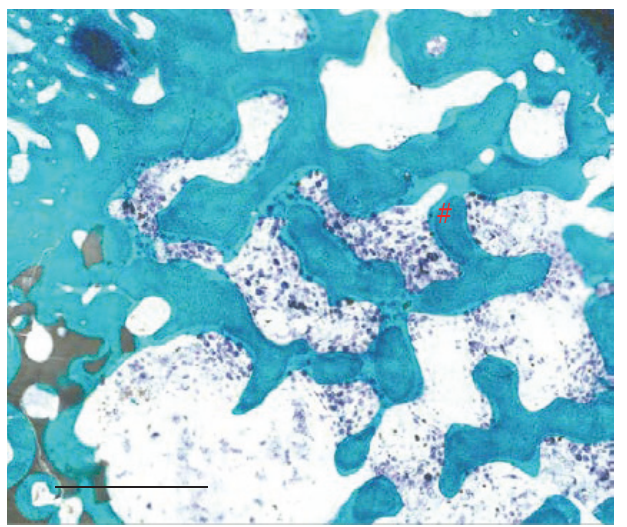

(f)

Figure 1: Representative histological images of the implantation site at 4 ((a), (c), and (e)) and 12 ((b), (d), and (f)) weeks from surgery. Bone was stained with Toluidine Blue, Acid Fuchsin, and Fast Green. The low magnification images reported in (a) and (b) allows distinguishing the bone area where nanoindentation has been carried out onto novel (*) and native (\#) tissue. Scale bar is $5 \mathrm{~mm}$ in (a) and (b) whereas it is $1 \mathrm{~mm}$ in $(\mathrm{c}-\mathrm{f})$. The newly formed bone as well as native bone is stained in light blue; brownish material is the residual macroporous HA scaffold.

contact depth $\left(h_{C}\right)$ value. Subsequently, $S$ and $A_{C}$ were used to compute $E_{R}$ and $H_{C}$ by OP equation [8]

$$
\begin{aligned}
E_{R} & =\frac{S \sqrt{\pi}}{2 \sqrt{A_{C}}}, \\
H_{C} & =\frac{P_{\mathrm{MAX}}}{A_{C}} .
\end{aligned}
$$

The reduced modulus is a combination of indenter tip and sample material properties. However, since bone $(E<$ $30 \mathrm{GPa}$ ) is by far less stiff than the diamond tip, the reduced modulus can be considered as the plane strain modulus which corresponds to the resistance to elastic deformation $\left(E_{R} \sim\right.$ $\left.E^{\prime}\right)$ [11]. In this model, bone is considered an elastoplastic material and its mechanical behavior during indentation 


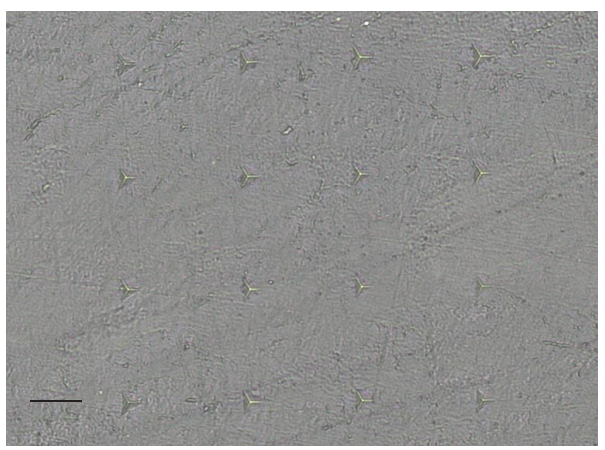

(a)

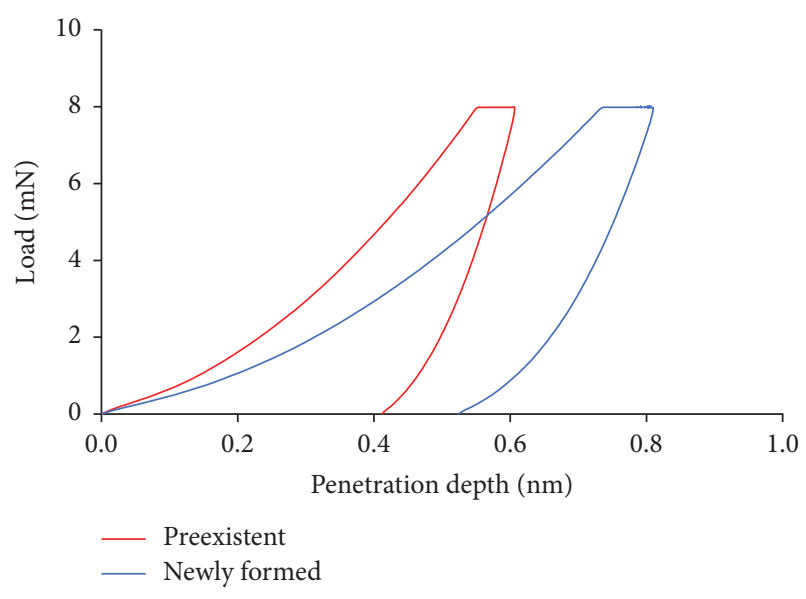

(b)

Figure 2: Nanoindentation test: (a) optical images of typically nanoindentation array of native bone. Scale bar is $10 \mu \mathrm{m}$. (b) Representative indentation curves for newly formed (blue) and preexistent (red) bone.

loading is modeled as purely elastic elements connected to a purely plastic one, in series as in the Maxwell combination. The hardness $(H)$ that represents the resistance to plastic deformation has been calculated based on Sakai expressions $[16,17]$. Briefly the resistance to the total deformation $\left(H_{C}\right)$ is the sum of the elastic element (characterized by $E^{\prime}$ ) and the plastic one (described by $H$ ):

$$
H_{C}=\frac{1}{\alpha_{1}\left(\left(\alpha_{2} E_{R}\right)^{-1 / 2}+\left(\alpha_{1} H\right)^{-1 / 2}\right)^{2}} \text {. }
$$

3.2. The Viscoelastoplastic (VEP) Model. Three rheological quadratic elements in series are considered in VEP model: a spring (elastic element; reversible process), a dashpot (viscous element; nonreversible, time-dependent process), and a slider (plastic element and nonreversible, time-independent process) [10]. The indentation viscosity $\left(\eta_{\mathrm{Q}}\right), E^{\prime}$, and $H$ are extracted from the displacement-time $(h-t)$ indentation curve by a three-step fitting process using the nonlinear least-square curve-fit function in MATLAB version 8.2.0.701 (MathWorks, Natick, MA). At first, $\eta_{\mathrm{Q}}$ was calculated by linear fit on the creep-hold period:

$$
\begin{aligned}
h^{\mathrm{CREEP}}(t)=\frac{\left(k t_{R}\right)^{1 / 2}}{\left(\alpha_{3} \eta_{\mathrm{Q}}\right)^{1 / 2}}\left(t-t_{1}\right)+h\left(t_{1}\right) & \\
& \\
& t_{R}<t<t_{R}+t_{C}
\end{aligned}
$$

where $\alpha_{3}=4.4$ is a dimensionless geometric constant for a perfect Berkovich indenter tip [10]; $k$ is the loading rate $\left(k=P_{\mathrm{MAX}} / t_{R}\right) ; t_{1}$ is defined as $t_{1}=t_{R}+t_{C} / 6$ to only consider the last $5 / 6$ of the holding segment and thus to obtain a better linear fit of the curve [12]; $t_{R}=10 \mathrm{~s}$ is the time rise; $t_{C}=$ $30 \mathrm{~s}$ is the creep time. Knowing $\eta_{Q}$, it is possible to obtain $E^{\prime}$ from fitting the unloading curve described by a viscoelastic behavior:

$$
\begin{aligned}
& h^{\mathrm{UNLOAD}}(t)=k^{1 / 2}\left(\frac{t_{R}^{3 / 2}-\left(2 t_{R}+t_{C}-t\right)^{3 / 2}}{3 / 2\left(\alpha_{3} \eta_{\mathrm{Q}}\right)^{1 / 2}}\right. \\
&\left.+\frac{\left(2 t_{R}+t_{C}-t\right)^{1 / 2}-t_{R}^{1 / 2}}{\left(\alpha_{2} E^{\prime}\right)^{1 / 2}}\right)+h^{\mathrm{CREEP}}\left(t_{R}+t_{C}\right), \\
& t_{R}>t_{R}+t_{C},
\end{aligned}
$$

where $\alpha_{2}=4.4$ [10]. The loading curve is instead described by viscoelastic-plastic behavior from which the hardness $H$ can be calculated according to

$$
\begin{array}{r}
h^{\mathrm{LOAD}}(t) \\
=(k t)^{1 / 2}\left(\frac{2 t}{3\left(\alpha_{3} \eta_{\mathrm{Q}}\right)^{1 / 2}}+\frac{1}{\left(\alpha_{2} E^{\prime}\right)^{1 / 2}}+\frac{1}{\left(\alpha_{1} H\right)^{1 / 2}}\right), \\
t<t_{R},
\end{array}
$$

where $\alpha_{1}=24.5[10]$.

The elastic element is characterized by $E^{\prime}$, the plastic one is characterized by the $H$ as previously described, and the viscous element is described by $\eta_{Q}$. The resistance to total deformation $H_{C}$ is finally obtained from

$$
\begin{aligned}
& H_{C} \\
& =\frac{1}{\alpha_{1}\left(2 t_{R} / 3\left(\alpha_{3} \eta_{Q}\right)^{-1 / 2}+\left(\alpha_{2} E^{\prime}\right)^{-1 / 2}+\left(\alpha_{1} H\right)^{-1 / 2}\right)^{2}} .
\end{aligned}
$$

3.3. Statistical Analysis. All data are presented as mean \pm standard deviation at a significant level of $p<0.05$. The 
Kolmogorov-Smirnov test was performed to test normality of the variables. Two-tailed paired Wilcoxon signed-rank was used to compare bone mechanical properties of OP and VEP models at 4 or 12 weeks. Besides, the Mann-Whitney $U$ test was used for the comparison of bone mechanical properties at different experimental times. The significance threshold was set at $p<0.05$.

The quality of fit is described by the fitting error index $\left(I_{2}\right)$ defined as

$$
\frac{\sqrt{\sum_{i=1}^{N}\left(y_{i}-y_{i}^{*}\right)^{2} / N}}{\sum_{i=1}^{N} y_{i}{ }^{*} / N},
$$

where $y_{i}$ are the experimental data, $y_{i}{ }^{*}$ are the fit data, and $N$ is the data numbers. In both models $I_{2}$ was calculated in the $40-98 \%$ region of the unloading curve.

All statistical analysis was performed using MATLAB version 8.2.0.701 (MathWorks, Natick, MA).

\section{Results}

Histological images of the regenerated condyle defects showed that a dense network of newly formed bone trabeculae was present at 4 weeks from surgery, both inside scaffold pores and in the peri-implant area (Figures 1(a), 1(c), and $1(\mathrm{e}))$. After 12 weeks, bone had undergone a significant remodeling process (Figures 1(b), 1(d), and 1(f)) as indicated by the lower amount of bone tissue within and around the implant, without compromising scaffold integration in the surrounding tissue.

The average fitting error index $I_{2}$ resulted $<0.1$ for both the models, indicating a good fitting of the experimental data (Figure 3). Noteworthy, the $I_{2}$ values of the VEP fitting ( 0.0044 at 4 weeks and 0.0041 at 12 weeks) were significantly lower than corresponding $I_{2}$ from the OP fitting $(0.043$ at 4 weeks 0.040 at 12 weeks) suggesting that the VEP model better describes the investigated tissue mechanics.

Representative load-penetration depth curves of newly formed and preexistent bone are shown in Figure 2(b) and the corresponding mechanical properties are reported in Figure 4. When comparing mechanical properties at the same experimental time point, values of native bone were higher than engineered ones suggesting that the regeneration process was still incomplete, also after 12 weeks [18]. Further, while observing the evolution of $E_{R}, H$, and $H_{C}$ with time, no significant differences were found between values at 4 and 12 weeks, independently from the bone tissue (engineered or native) and model (OP or VEP) investigated. The OP model was found to underestimate $E_{R}$ while overestimating $H$ compared to the VEP model, while $H_{C}$ was seemed not to be affected. Interestingly, the gap between the mechanical properties of engineered and native bone was larger for the VEP model $\left(+21.4 \%\right.$ for $E_{R} ;+20.9 \%$ for $H_{C}$ and $+19.5 \%$ for $H)$ compared to the OP model $\left(+15.8 \%\right.$ for $E_{R} ;+16.5 \%$ for $H_{C}$ and $+15.5 \%$ for $H$ ).

Noteworthy, contrary to the OP model, the VEP model allowed the additional analysis of the evolution of viscous content of bone during the regeneration process (Figure 5). In particular, data indicated that whereas the viscosity of native bone was similar between 4 and 12 weeks, the viscosity of newly formed bone trabeculae significantly increased (+34\%) during bone healing, till achieving a similar value to that of preexisting bone.

\section{Discussion}

In this study we investigated the mechanical properties of newly formed bone tissue grown within the pores of a hydroxyapatite (HA) macroporous scaffolds implanted in rabbit femoral condyles, with the final aim of comparing the ability of OP and VEP models to provide information over bone regeneration from a micro/nanomechanical point of view.

The results reported here clearly highlighted a higher sensitivity of the VEP model to determine the mechanical parameters of bone tissue with respect to the OP model as indicated by the higher gap between the mechanical properties of engineered and native bone found with the former method. Furthermore, the possibility of following the evolution of $\eta_{Q}$ during bone healing provided by the VEP model may allow obtaining additional insights above bone tissue maturation not achievable by the OP model.

To date, mechanical properties of engineered bone have been almost exclusively assessed with the OP model [19]. Some reasons for the popularity of OP include (i) tradition and precedent; it was the first model established; (ii) OP which is programmed into the software provided with commercially available indenter systems, (iii) oversimplification of bone material and behaviors, (iv) a lack of insight by authors who fail to recognize the limitations of the OP approach, and (v) the OP model which provides a reasonable baseline estimation of bone properties. Typically, values of $E_{R}$ and $H_{C}$ ranging from 16 to $22 \mathrm{GPa}$ and from 0.6 to $1.2 \mathrm{GPa}$, respectively, have been reported [20-22]. Data obtained in our study applying the OP model well fit within these range. Only one study implementing a first-version of VEP addressing the bone healing around an implant is present [23]. However, according to this preliminary version of the model, the creep-hold period was not included in the indentation profile, hindering the discerning of the viscous contribution. Instead, we carried out in this study for the first time the comparison between mechanical properties of newly formed and preexistent bone taking advantage of the full expression of the VEP model (as reported above), in such a way considering all the deformation modes on the tissue.

Generally, four modes of deformation can be elicited during indentation testing: elastic, plastic, viscous, and fracture [9]. Typically, bone tissue is tested with a sharp (i.e., Berkovich) indenter tip, which tends to deform it in a viscoelastic-plastic manner [10]. Interestingly, literature reports that each different contribution (i.e., plastic, elastic, and viscous) to total deformation can be correlated to specific histological properties of bone tissue. In particular, $E_{R}$ and $H_{C}$ values are influenced by the amount of mineral phase, as demonstrated through backscattered electron imaging (qBEI) [24] and Fourier transform infrared spectroscopy (FTIR) [25] studies. Bala et al. showed that $H$ (calculated by OP) can be instead related to the degree of maturity of collagen fibers [25]. Finally, several studies exploiting different 


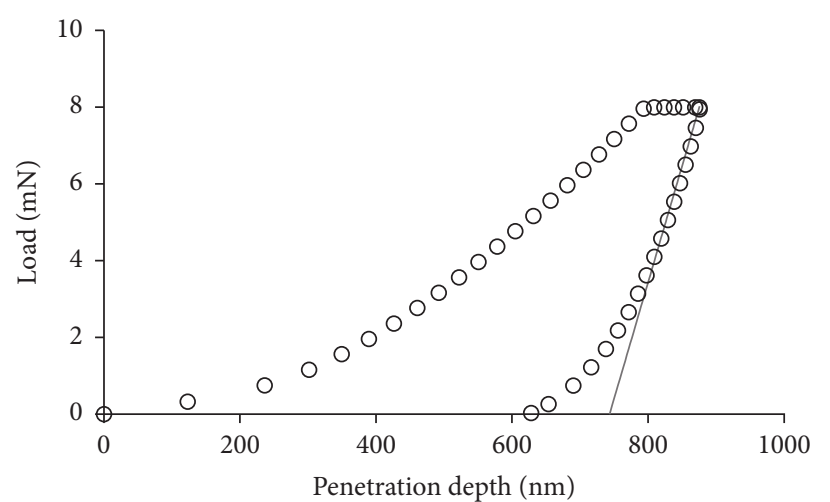

- Nanoindentation data OP fit

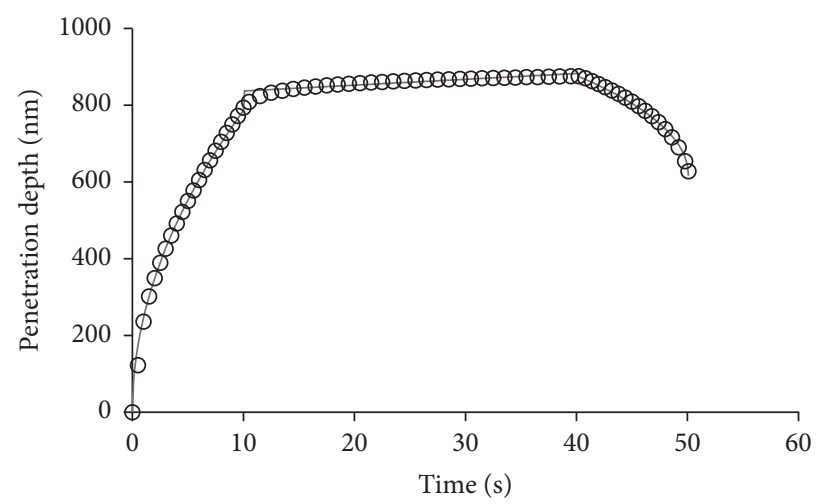

O Nanoindentation data - VEP fit

(a)

(b)

FIGURE 3: Representative load-displacement (a) and displacement-time (b) nanoindentation plots fitted with the OP and VEP methods, respectively. Fitting: grey solid line. Experimental data: round markers.

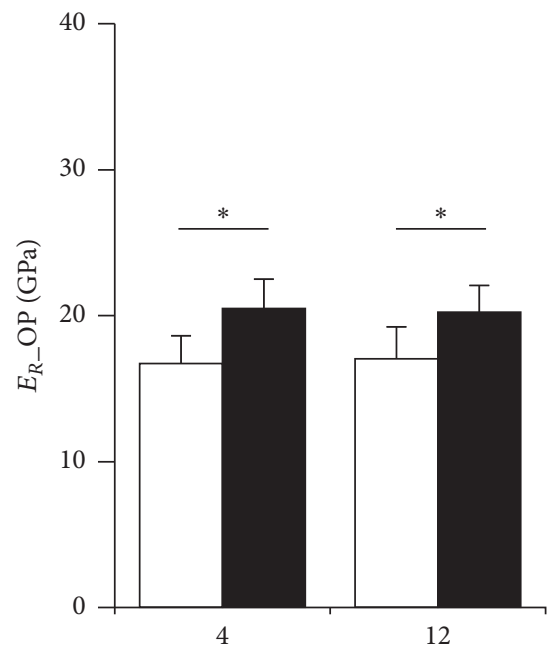

(Weeks)

(a)

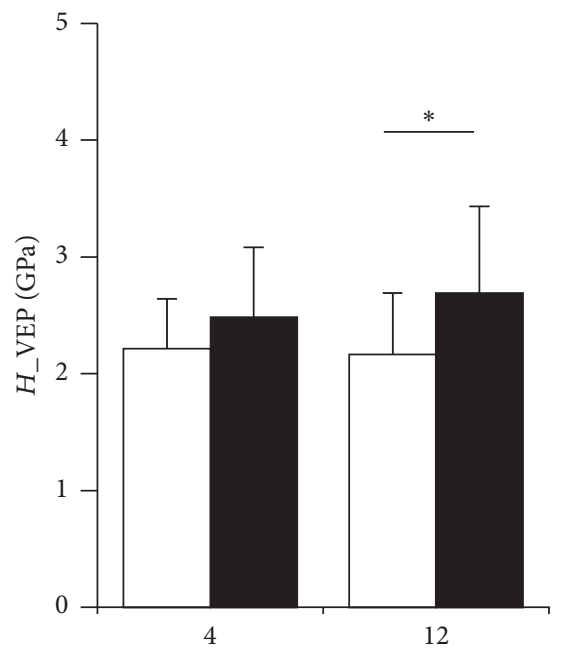

(Weeks)

(d)

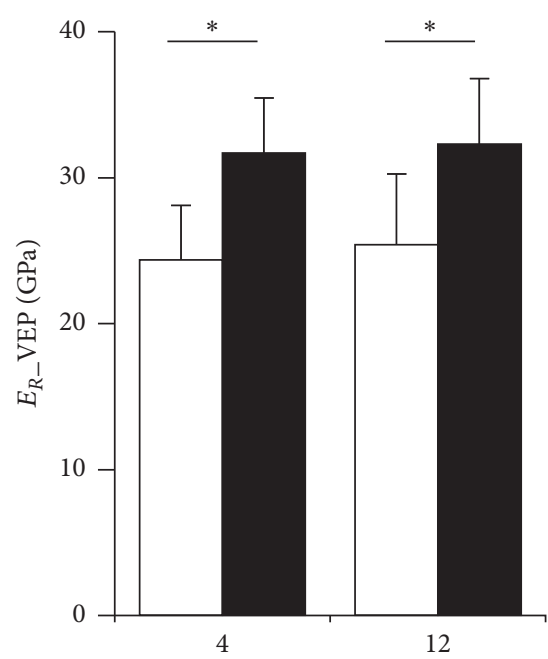

(Weeks)

(b)

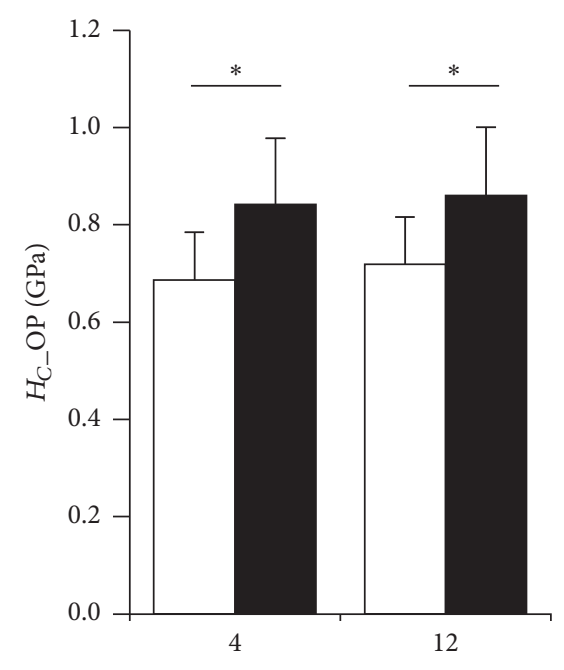

(Weeks)

(e)

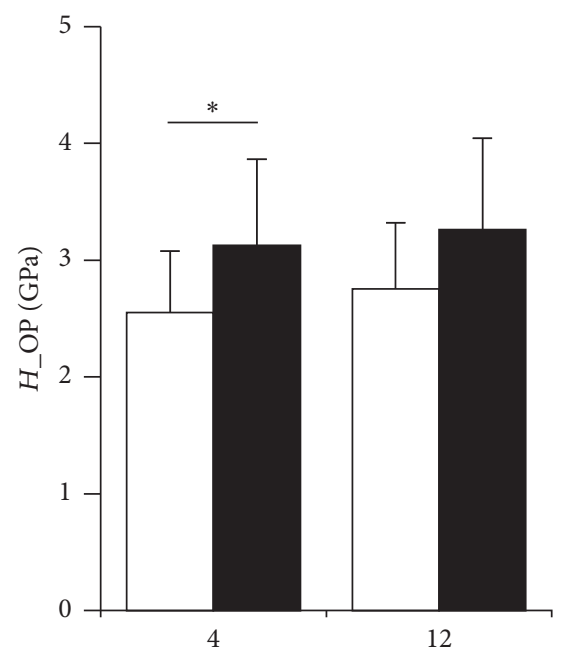

(Weeks)

(c)

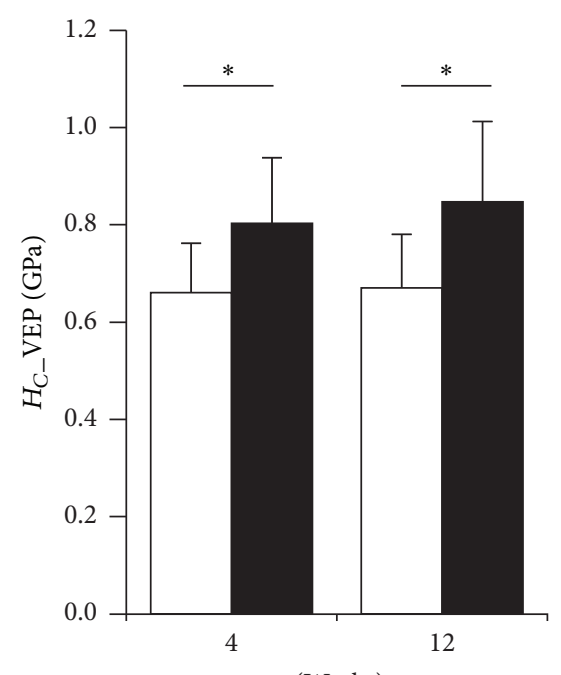

(Weeks)

(f)

Figure 4: Elastic modulus $\left(E_{R}\right.$, (a) and (b)), hardness $(H,(c)$ and $(d))$, and contact hardness $\left(H_{C}\right.$, (e) and (f)) of engineered (white) and preexistent (black) bone obtained by implementing the OP ((a), (c), and (e)) or VEP ((b), (d), and (f)) model. $*$ refers to $p<0.05$. 


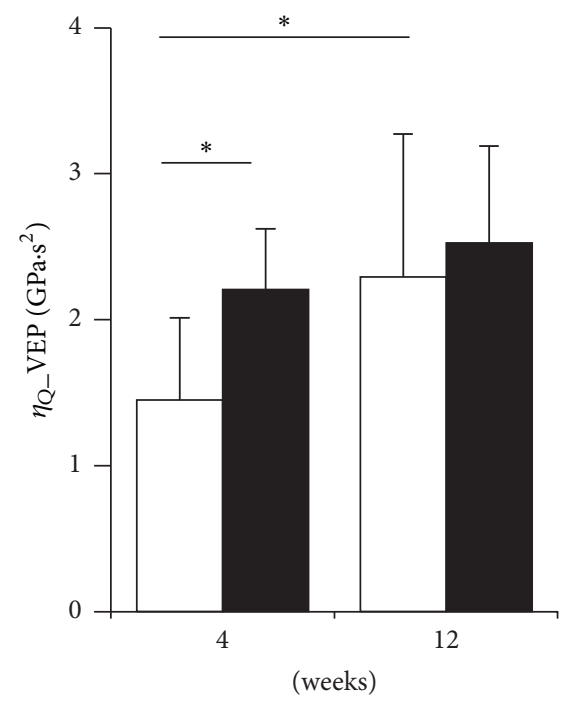

FIGURE 5: Viscosity index $\left(\eta_{Q}\right)$ of engineered (white) and native (black) bone tissue, as obtained by VEP analysis. * refers to $p<0.05$.

viscoelastic models highlighted a correlation between $\eta_{Q}$ and collagen interfibril sliding motions [26, 27].

The main limitation of this study is related to the sample preparation method (dehydration, fixation, and PMMAembedding). This preparation is known to generally lead to higher $E_{R}$ values when investigating fresh bone tissue $[12,13]$. However, it is a common practice to use the same samples for multidisciplinary testing (such as histological and histomorphometrical analysis) that necessitates the PMMAinclusion [21]. Further, all samples were prepared following the same protocol and this should not affect comparative trends [13]. Another limitation may represented by the use the sharp Berkovich tip, as it is known that it is not able to elicit the viscosity deformation than does a spherical tip. Nevertheless, the Berkovich tip was used in this study in order to analyze small volumes within the thin bone trabeculae obtained according to the in vivo experimental study. Also, VEP assumes the material is viscoelastoplastic with a linear creep rate which would be more accurately modeled by a decaying, nonlinear creep rate [11]. The VEP is a single time constant model and its prediction capability is limited when indenting a hierarchical material with different time scales such as bone. Nevertheless, VEP is useful for comparison of groups within studies [12] which was our objective more than perfectly determining quantitative material properties. Finally, another limitation is related to the use of the HA macroporous scaffolds: even if it is known to promote extensive bone tissue regeneration [28], the 3D geometry of the scaffold could influence the mechanical properties of regenerated bone trabeculae compared to other models, and this should be taken into account when comparing the outcomes of different nanoindentation studies.

Concluding, by means of nanoindentation analysis we demonstrated that the regenerative process was still far from complete maturation, as indicated by the gap between mechanical properties of native and engineered tissue, wider for the VEP model than OP. Noteworthy, the VEP highlighted a progression towards the native tissue adding the consideration of viscosity. Interestingly, the OP model would have suggested an almost concluded remodeling process. Thus, the results reported in this work highlight the better ability of VEP model into providing important insights over bone regeneration process, by allowing deepening the bone remodeling phenomena in terms of structural material properties by the evidence of a broader spectrum of deformation components.

\section{Conclusions}

The Oliver and Pharr method is by far the most implemented model when analyzing indentation data. As completely mineralized, mature bone tissue is not highly viscoelastic and $\mathrm{OP}$ may give a reasonable estimate of its stiffness and hardness. However, for biomaterials that exhibit a substantial viscoelasticity component, such as newly formed bone, the OP model fails to correctly assess their mechanical behavior. In these cases, the VEP model should be preferred. Indeed, the results reported in this work highlight the better ability of VEP model into providing important insights over bone regeneration process, by allowing deepening the bone remodeling phenomena in terms of structural material properties by the evidence of a broader spectrum of deformation components.

\section{Conflicts of Interest}

The authors have no conflicts of interest to disclose.

\section{Acknowledgments}

This study was partially supported by the FP7 European Project "Magnetic Scaffolds for In Vivo Tissue Engineering" (NMP3-LA-2008-214686) and by the " $5 \times 1000$ " (2013) funding, provided by Istituto Ortopedico Rizzoli, Bologna (Italy).

\section{References}

[1] S. Weiner and H. D. Wagner, "The material bone: structuremechanical function relations," Annual Review of Materials Science, vol. 28, no. 1, pp. 271-298, 1998.

[2] N. Reznikov, R. Shahar, and S. Weiner, "Bone hierarchical structure in three dimensions," Acta Biomaterialia, vol. 10, no. 9, pp. 3815-3826, 2014.

[3] D. M. Ebenstein and L. A. Pruitt, "Nanoindentation of biological materials," Nano Today, vol. 1, no. 3, pp. 26-33, 2006.

[4] C. Wang, Y. Wang, A. Wang, Q. Guo, J. Peng, and S. Lu, "Application of the nanoindentation technique in bone micromechanics," Journal of Computational and Theoretical Nanoscience, vol. 12, no. 11, pp. 4005-4009, 2015.

[5] L. Feng, M. Chittenden, J. Schirer, M. Dickinson, and I. Jasiuk, "Mechanical properties of porcine femoral cortical bone measured by nanoindentation," Journal of Biomechanics, vol. 45, no. 10, pp. 1775-1782, 2012.

[6] S. Hengsberger, A. Kulik, and P. H. Zysset, "Nanoindentation discriminates the elastic properties of individual human bone lamellae under dry and physiological conditions," Bone, vol. 30, no. 1, pp. 178-184, 2002. 
[7] P. K. Zysset, X. E. Guo, C. E. Hoffler, K. E. Moore, and S. A. Goldstein, "Elastic modulus and hardness of cortical and trabecular bone lamellae measured by nanoindentation in the human femur," Journal of Biomechanics, vol. 32, no. 10, pp. 1005-1012, 1999.

[8] W. C. Oliver and G. M. Pharr, "Measurement of hardness and elastic modulus by instrumented indentation: advances in understanding and refinements to methodology," Journal of Materials Research, vol. 19, no. 1, pp. 3-20, 2004.

[9] M. L. Oyen and R. F. Cook, "A practical guide for analysis of nanoindentation data," Journal of the Mechanical Behavior of Biomedical Materials, vol. 2, no. 4, pp. 396-407, 2009.

[10] M. L. Oyen and R. F. Cook, "Load-displacement behavior during sharp indentation of viscous-elastic-plastic materials," Journal of Materials Research, vol. 18, no. 1, pp. 139-150, 2003.

[11] S. E. Olesiak, M. L. Oyen, and V. L. Ferguson, "Viscous-elasticplastic behavior of bone using Berkovich nanoindentation," Mechanics of Time-Dependent Materials, vol. 14, no. 2, pp. 111124, 2010.

[12] N. Rodriguez-Florez, M. L. Oyen, and S. J. Shefelbine, "Insight into differences in nanoindentation properties of bone," Journal of the Mechanical Behavior of Biomedical Materials, vol. 18, pp. 90-99, 2013.

[13] A. J. Bushby, V. L. Ferguson, and A. Boyde, "Nanoindentation of bone: Comparison of specimens tested in liquid and embedded in polymethylmethacrylate," Journal of Materials Research, vol. 19, no. 1, pp. 249-259, 2004.

[14] Y.-T. Cheng, W. Ni, and C.-M. Cheng, "Determining the instantaneous modulus of viscoelastic solids using instrumented indentation measurements," Journal of Materials Research, vol. 20, no. 11, pp. 3061-3071, 2005.

[15] B. J. Briscoe, L. Fiori, and E. Pelillo, "Nano-indentation of polymeric surfaces," Journal of Physics D: Applied Physics, vol. 31, no. 19, pp. 2395-2405, 1998.

[16] M. L. Oyen, "Nanoindentation hardness of mineralized tissues," Journal of Biomechanics, vol. 39, no. 14, pp. 2699-2702, 2006.

[17] M. Sakai, "Meyer hardness: A measure for plasticity?" Journal of Materials Research, vol. 14, no. 9, pp. 3630-3639, 1999.

[18] M. Bianchi, M. Boi, M. Sartori et al., "Nanomechanical mapping of bone tissue regenerated by magnetic scaffolds," Journal of Materials Science. Materials in Medicine, vol. 26, no. 1, p. 5363, 2015.

[19] K. Tai, G. Pelled, D. Sheyn et al., "Nanobiomechanics of repair bone regenerated by genetically modified mesenchymal stem cells," Tissue Engineering Part A, vol. 14, no. 10, pp. 1709-1720, 2008.

[20] E. Arrigoni, L. De Girolamo, A. Di Giancamillo et al., "Adiposederived stem cells and rabbit bone regeneration: histomorphometric, immunohistochemical and mechanical characterization," Journal of Orthopaedic Science, vol. 18, no. 2, pp. 331-339, 2013.

[21] A. Cipitria, C. Lange, H. Schell et al., "Porous scaffold architecture guides tissue formation," Journal of Bone and Mineral Research, vol. 27, no. 6, pp. 1275-1288, 2012.

[22] G. Pelled, K. Tai, D. Sheyn et al., "Structural and nanoindentation studies of stem cell-based tissue-engineered bone," Journal of Biomechanics, vol. 40, no. 2, pp. 399-411, 2007.

[23] M. L. Oyen and C.-C. Ko, "Examination of local variations in viscous, elastic, and plastic indentation responses in healing bone," Journal of Materials Science: Materials in Medicine, vol. 18, no. 4, pp. 623-628, 2007.
[24] H. S. Gupta, S. Schratter, W. Tesch et al., "Two different correlations between nanoindentation modulus and mineral content in the bone-cartilage interface," Journal of Structural Biology, vol. 149, no. 2, pp. 138-148, 2005.

[25] Y. Bala, B. Depalle, T. Douillard et al., "Respective roles of organic and mineral components of human cortical bone matrix in micromechanical behavior: An instrumented indentation study," Journal of the Mechanical Behavior of Biomedical Materials, vol. 4, no. 7, pp. 1473-1482, 2011.

[26] I. L. Jäger, "A model for the stability and creep of organic materials," Journal of Biomechanics, vol. 38, no. 7, pp. 1459-1467, 2005.

[27] D.-G. Kim, S. S. Huja, H. R. Lee, B. C. Tee, and S. Hueni, "Relationships of viscosity with contact hardness and modulus of bone matrix measured by nanoindentation," Journal of Biomechanical Engineering, vol. 132, no. 2, pp. 024502-024506, 2010.

[28] S. V. Dorozhkin, "Bioceramics of calcium orthophosphates," Biomaterials, vol. 31, no. 7, pp. 1465-1485, 2010. 

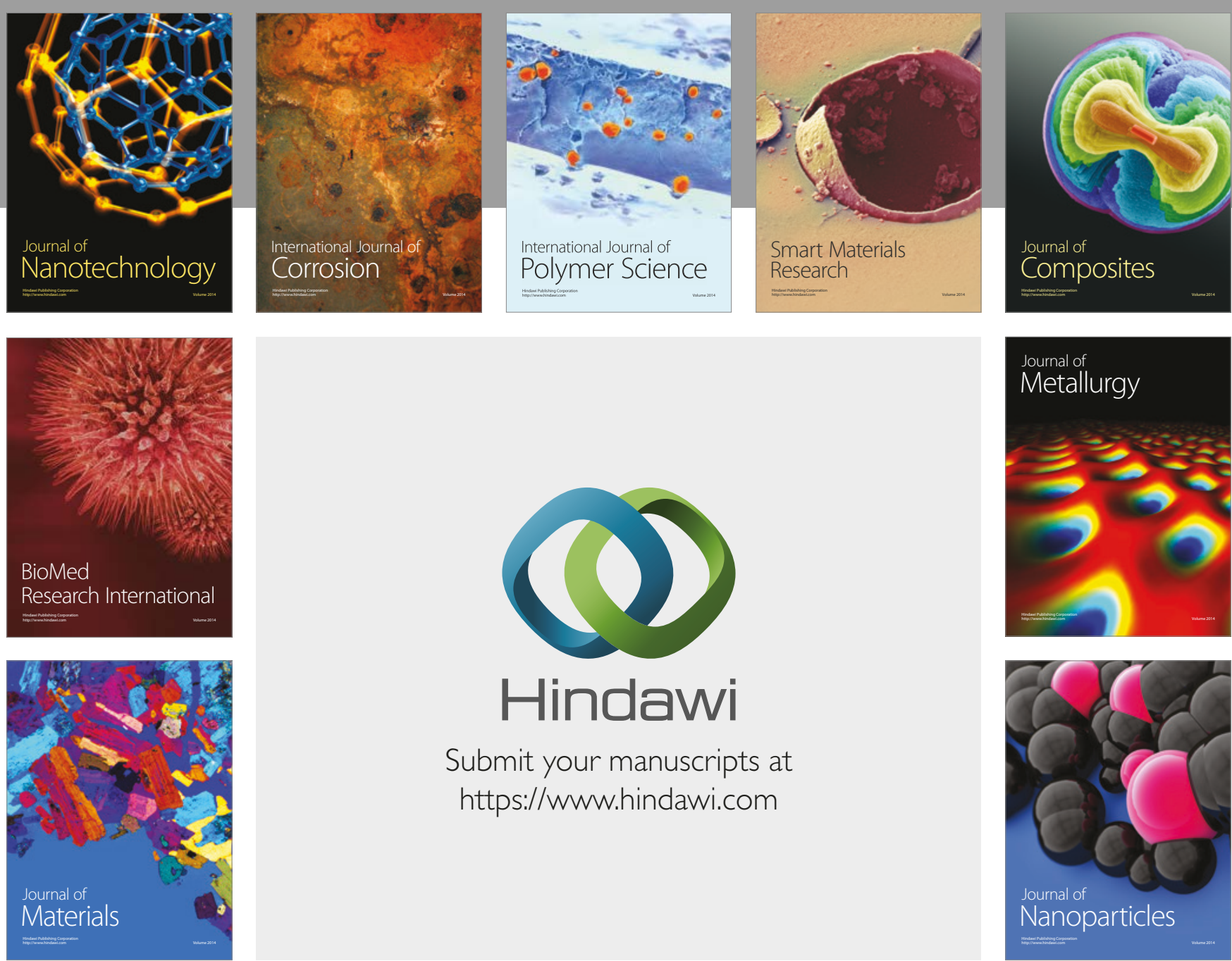

\section{Hindawi}

Submit your manuscripts at

https://www.hindawi.com
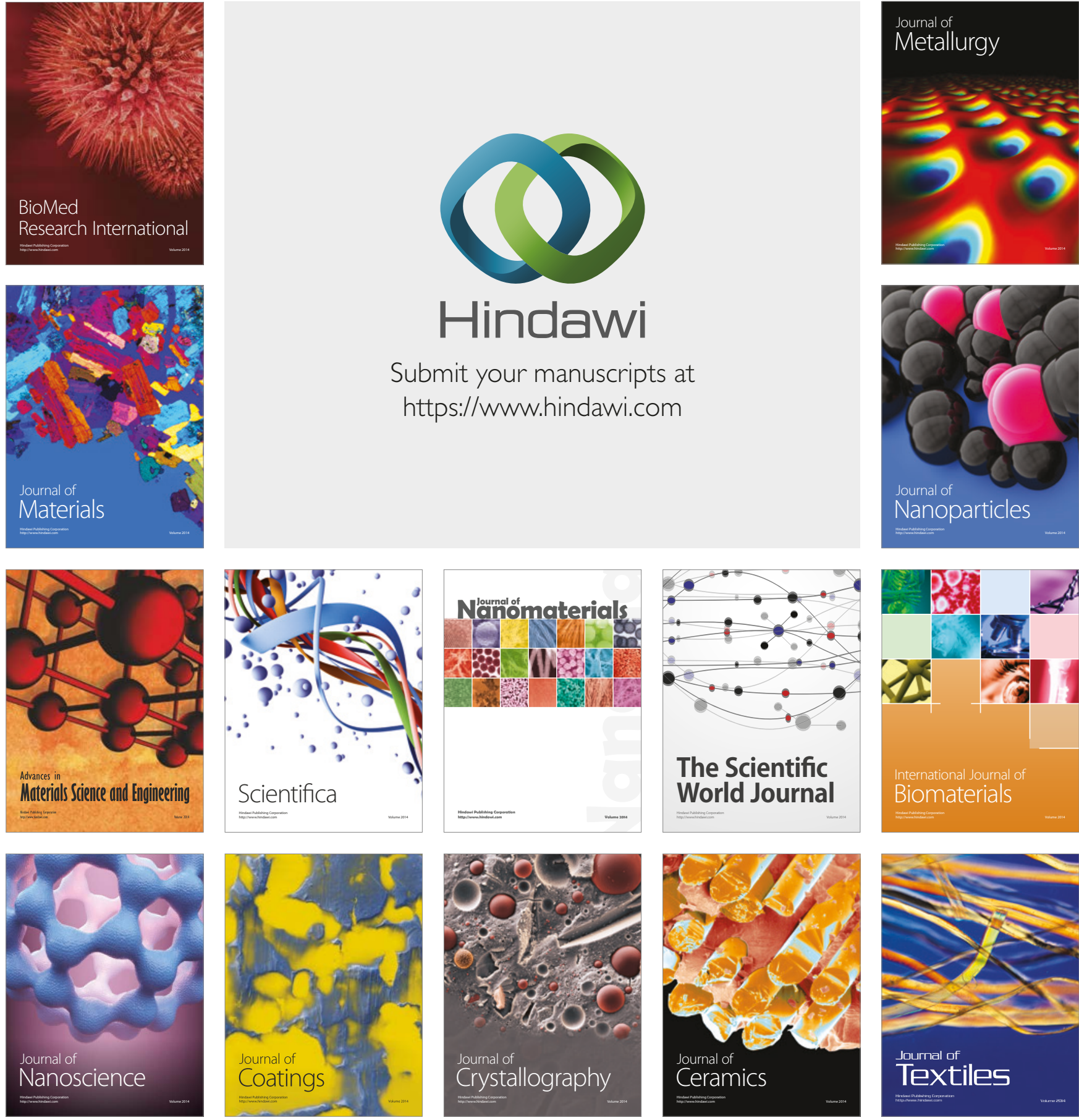

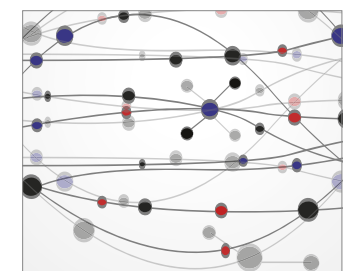

The Scientific World Journal
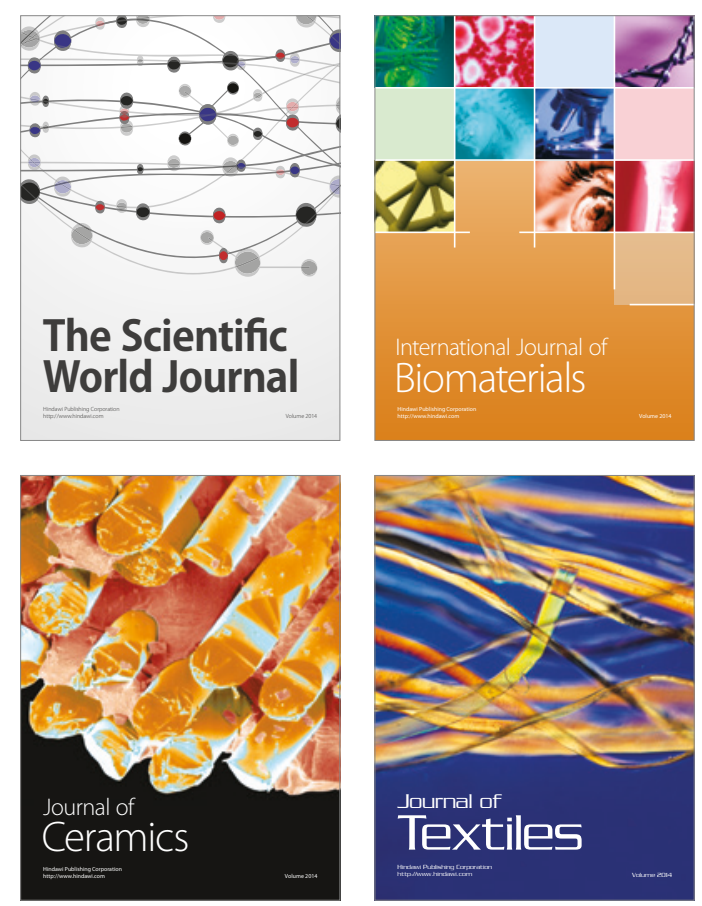